\title{
Testimonios, discurso experto y comisiones de la verdad: el contexto de la denuncia
}

\author{
Testimonies, expert discourse and truth commissions: \\ the context of denunciation
}

\author{
Silvia Rodríguez MAEso \\ Centro de Estudos Sociais - CES \\ Universidade de Coimbra \\ srodrig@ces.uc.pt
}

Recibido: 10.11.2010

Aprobado definitivamente: 03.06.2011

\section{RESUMEN}

Este texto analiza las Comisiones de la Verdad como mecanismos políticos desde una doble perspectiva: uno, los testimonios como elemento central que otorga legitimidad ético-política a las comisiones así como por su labor en la determinación de las violaciones de los derechos humanos; y dos, el trabajo de los profesionales del derecho y de las ciencias sociales como expertos que aportan legitimidad científica a estos procesos y que son también testigos privilegiados de la producción de la verdad. A través del estudio de un caso - la Comisión de la Verdad y Reconciliación peruana — se discuten las posibilidades del conocimiento científico para comprender de forma más completa los "hechos de violencia" así como la necesidad de problematizar ciertos presupuestos interpretativos en relación a la agencia política de determinadas poblaciones, en este caso, de las comunidades campesinas andinas.

Palabras clave: Comisiones de la verdad, testimonios, discurso experto, verdad jurídica/histórica, Perú.

\begin{abstract}
This text analyses Truth Commissions as a political mechanism engaging with two main questions: one, the central role of testimonies in providing both ethical and political legitimacy to truth commissions and information regarding the violation of human rights; two, the work of professionals from law and the social sciences in providing scientific legitimacy and in their position as 'privileged' witnesses of the truth-telling process. Throughout the analysis of a specific case - the Peruvian Truth and Reconciliation Commission - I discuss the possibilities of scientific knowledge to produce a more complete understanding of "the violence" and the need to question certain interpretative assumptions regarding the political agency of certain populations, in this case, of the Andean rural communities.
\end{abstract}

Keywords: Truth Commissions, Testimonies, Expert Discourse, Judicial / Historical Truth, Peru. 


\section{SUMARIO}

1. Verdades parciales y tecnologías del testimonio: el derecho y las ciencias sociales en la CVR peruana. 2. Verdad "jurídica" e "histórica": los contornos políticos de una posible crisis de representación. 3. La política de lo silenciado y de lo implícito. 4. Testimonios, poder y conocimiento científico. 5. ¿A quién se dirigió la CVR peruana?: los límites de la legitimidad científica. 
El Comité de Violaciones de los Derechos Humanos [de la CVR sudafricana] invitó a las personas a producirdeclaraciones escritas y orales. Aproximadamente un diez por ciento fueron escuchadas en audiencias públicas organizadas por todo el país. Episodios seleccionados o partes de los testimonios públicos fueron transmitidos por televisión y radio. La Comisión creó la oportunidad de observar y de ser testigo de un evento de recordación: la duplicación de la figura de testigo [witness] (Ross, 2001: 253). ${ }^{1}$

[El Informe Final de la CVR peruana] es una utilización de la información de acuerdo a tus hipótesis y entonces ahí hay un tema porque en realidad, ¿es la voz de los 17.000 testimonios o es la lectura de los 17.000 testimonios?; entonces, por tanto, para mí el informe de la CVR, como yo siempre digo, el informe de la CVR es un punto de partida, es una narración, como puede haber otras, pero hacer que el informe de la CVR se convierta en la Biblia (...) es el peor error que podemos cometer porque significa dejar de lado las discrepancias, dejar de lado a aquellos que no están de acuerdo, dejar de lado a aquella gente que dijo "ese testimonio no me representa", gente que no habló. Entonces hay la concepción de una verdad, de una narración construida por una comunidad intelectual, entonces, que esa comunidad intelectual se haya basado en 17.000 testimonios, ¿le da legitimidad?, eso es discutible; digamos, finalmente, sigue siendo una comunidad intelectual analizando un proceso (Abogada, entrevista de la autora; Lima: septiembre 2006).
Las Comisiones de la Verdad (CV) se han convertido, desde mediados de la década de 1980, en "estructuras estandarizadas" (Wilson, 2001: XVIII) para dar cuenta desde el Estado de la violación sistemática de derechos humanos mediante la investigación de los hechos y las responsabilidades correspondientes. Las CV se insertan por tanto entre los mecanismos con mayor relevancia dentro del proceso de globalización del discurso y práctica jurídica de los derechos humanos, así como del campo de la llamada "justicia transicional" que analiza los contextos de cambio político de un régimen totalitario hacia uno democrático-liberal, y en especial, el papel de la justicia y del derecho. ${ }^{2}$

En este texto voy a centrarme en dos aspectos interrelacionados del trabajo de las CV: uno, los testimonios como elemento central de las CV tanto desde el punto de vista de su legitimidad ético-política como de su labor en la determinación de las violaciones de los derechos humanos; y dos, el trabajo de los profesionales del derecho y de las ciencias sociales dentro de las CV como expertos que aportan legitimidad científica a estos procesos y que son también testigos privilegiados de la producción de la verdad, en esa duplicación de la figura del testigo que las comisiones propician (Ross, 2003: 253). Mi análisis se construye a partir de una investigación sobre la Comisión de la Verdad y Reconciliación peruana (CVR-P), ${ }^{3}$ centrada en la experiencia de diferentes profesionales que en ella trabajaron, así como en el análisis de documentos de trabajo que la comisión produjo durante el período de su mandato (2001-2003). ${ }^{4}$ No obstante, no pretende ofrecer una evaluación del trabajo de las $\mathrm{CV}$ en un sentido

\footnotetext{
${ }^{1}$ Todas las traducciones son propias salvo especificación.

${ }^{2}$ Sobre el trabajo y repercusión de las comisiones de la verdad en diferentes contextos de democratización del sistema político, véase, entre otros: Allen (1999); Barahona de Brito (1997); Castillejo (2007); Cavallaro y Albuja (2008); Crenzel (2009); Hayner (2001); Minow (1998); Popkin y Roht-Arriaza (1995); Rotberg y Thompson (2000); Steiner (1997); Teitel (2000); Wilson (2001).

${ }^{3}$ La Comisión de la Verdad fue establecida en el año 2000 durante el gobierno de transición presidido por Valentín Paniagua tras la caída del régimen de Alberto Fujimori (1990-2000). Cuando Alejandro Toledo gana las elecciones presidenciales ratificó y complementó su designación como Comisión de la Verdad y Reconciliación. La CVR-P tenía como finalidad "esclarecer el proceso, los hechos y responsabilidades de la violencia terrorista y de la violación a los derechos humanos producidos desde mayo de 1980 hasta noviembre de 2000, imputables tanto a las organizaciones terroristas como a los agentes del Estado, así como proponer iniciativas destinadas a afirmar la paz y la concordia entre los peruanos" (Decretos Supremo 065-2001-PCM).

${ }^{4}$ El trabajo empírico se centró por un lado, en entrevistas a profesionales de las ciencias sociales y del derechos que trabajaron en diferentes ámbitos y niveles de la CVR-P (comisionados, área jurídica, estudios en profundidad, área metodológica, estudios de caso, redacción del informe final, toma de testimonios en los equipos móviles), y por otro lado, el análisis de documentación producida durante el trabajo de la CVR y que se encuentra reunida en el archivo de la Defensoría del Pueblo en Lima (Centro de información para la Memoria Colectiva y los Derechos Humanos). Realicé el trabajo empírico entre los meses de marzo-abril y agosto-noviembre de 2006, período en el cual fui investigadora asociada en el Instituto de Estudios Peruanos (IEP).
} 
global. Las CV son procesos complejos y no deben ser consideradas como totalidades monolíticas. En este sentido, lo que pretendo es mostrar - desde el estudio de los dos aspectos ya mencionados- la multiplicidad de perspectivas de los expertos, los encuentros y desencuentros entre ellas, teniendo en cuenta los diferentes niveles en que trabajaron: desde el nivel más micro de la toma de testimonios y de los estudios en profundidad de los casos estudiados, hasta el nivel más macro de construcción del marco jurídico-legal y de la meta-narrativa que unifican el Informe Final (IF). Más concretamente, me interesa discutir las posibilidades de intervención del conocimiento científico en la interpretación de los "hechos de violencia", que pasa por mostrar cómo la problematización de la agencia política de determinadas poblaciones, en este caso de las comunidades campesinas andinas, continúa siendo un aspecto esencial para comprender las relaciones de poder que atraviesan procesos como las $\mathrm{CV}$.

Sitúo el trabajo de los expertos considerando las $\mathrm{CV}$ como procesos que siguen una lógica representacional y establecen lo que Luc Boltanski (1990; 2007) denomina un "régimen de justicia". Así, la CVR-P y en particular el trabajo científico que lo respaldó, se desarrolló dentro de un régimen representacional donde las operaciones principales son la denuncia pública — que fija y legitima la condición de víctimas - y la acusación - que refiere a la revelación de las acciones e intenciones de los perpetradores-. De ese modo considero que las CV son procesos políticos que tratan de evitar una crisis en la representación de las violaciones a los derechos humanos, es decir, de evitar no solo que las operaciones de acusación y de denuncia se estabilicen en una situación de precariedad - en la que sea imposible diferenciar entre perpetradores y víctimas (y sus respectivas responsabilidades en los hechos)-, sino que incluso estos roles se intercambien constantemente. La CVR-P no solamente debió lidiar con esa "zona gris" descrita por Primo Levi para referirse a aquellas situaciones e individuos que no pueden ser fácilmente identificables en base al binomio víctimas/verdugos, sino también con una suerte de círculo vicioso donde quien es considerado perpetrador en un determinado contexto y desde una determinada perspectiva, pasa a ser víctima en otro. Es decir, estaríamos ante una constante "incertidumbre referencial" (Boltanski, 2007: 300-305), que revelaría, entre otras cosas, una lucha ideológica dentro del análisis e interpretación de los hechos, y sobre todo de sus causas y principales consecuencias, incluyendo los criterios que definen el estatus de perpetrador y de víctima. Frente a esta incertidumbre, considero que la CVR-P fue parte del proceso de delineamiento de los contornos políticos del conflicto armado y de sus causas, contribuyendo a definir el marco interpretativo de "la violencia" y de su historicidad.

El texto se divide en cinco secciones. En la primera me centro en cómo los testimonios se encuadraron en las diferentes "tecnologías de la verdad" (Wilson, 2001: 33) que la maquinaria de una CV pone en funcionamiento, teniendo en cuenta la labor de los expertos en derecho y en ciencias sociales. En la segunda sección analizo las estrategias interpretativas que los profesionales del derecho y las ciencias sociales asumieron para gestionar los contornos políticos que han moldado la producción de los testimonios sobre "la violencia". La tercera está dedicada a comprender el estatus de los testimonios como narrativas reveladoras del contexto de la denuncia a partir de la negociación con lo silenciado y con lo implícito. En la cuarta sección discuto lo que podemos esperar del trabajo de los científicos sociales en un proceso como el de las CV así como en el contexto que las sucede. Finalmente, concluyo con unas reflexiones en torno a los límites de la legitimidad científica en procesos como las CV a partir de la forma como construye a sus audiencias privilegiadas.

\section{VERDADES PARCIALES Y TECNOLOGÍAS DEL TESTIMONIO: EL DERECHO Y LAS CIENCIAS SOCIALES EN LA CVR PERUANA}

Analizar cómo se establece un régimen de justicia desde un proceso como una comisión de la verdad requiere tener en cuenta ciertas cuestiones: ¿estamos ante un tipo de verdad específico y ya consolidado?, ¿cómo se inserta en procesos ya en marcha en contextos afectados por conflictos? Mahmood Mamdani, en su análisis de la comisión sudafricana, consideró dos tipos de verdad: la verdad que se enfrenta con el poder para mostrar públicamente tensiones irresueltas, y la verdad institucional, que en vez de oponerse al poder se vincula con él, ofreciendo una "versión parcial" de los hechos. Para 
Mamdani la verdad "producida institucionalmente" de la CVR sudafricana se estableció a partir de la experiencia de una "reducida minoría (...) que incluía dos grupos, por un lado, los perpetradores - los agentes estatales - y por el otro, las víctimas - los activistas políticos-. La CVR sudafricana definió a más de 20.000 sudafricanos como las 'víctimas' del apartheid, dejando a la gran mayoría en el frío proverbial" (2000: 178). Si bien Mamdani trabaja a partir de las características del contexto sudafricano y concretamente del apartheid como régimen, creo que su énfasis en la producción de una "verdad institucional" como característica del trabajo de la comisión y su relación con la diferenciación entre víctimas y perpetradores son útiles para comprender el proceso de la CVR-P.

La legitimidad de la verdad producida en la CVR-P se confió, en gran medida, a la labor científica de profesionales del derecho y las ciencias sociales, y en particular, a su capacidad de trabajar con las informaciones provenientes de los testimonios y de los estudios de caso:

Muchas de las investigaciones llevadas a cabo por la CVR han tenido por finalidad establecer un registro detallado y preciso de los hechos de violencia, de las condiciones en que se produjeron, de sus participantes directos $y$ de las secuelas que dejaron. En todos estos casos, la CVR recurrió a los expertos y a los métodos científicos y técnicos más actualizados, a fin de garantizar la mayor objetividad posible: informes criminológicos, pericias judiciales, antropología forense, análisis de laboratorio, etc. Pero esta dimensión cientifica ha estado presente igualmente en los trabajos de análisis e interpretación de las causas de los hechos, pues se solicitó el concurso de una amplia gama de cientificos que, desde disciplinas distintas - como la antropología, la sociología, la historia y la sicología - y con el instrumental metodológico disponible, han contribuido a esclarecer el proceso en cuestión. Se ha dado asi un respaldo científico al relato éticamente articulado (CVR, 2003, Introducción: 32-33).

Es en este proceso de legitimación del trabajo de la CVR-P y su relación directa con las narrativas recogidas en los testimonios que considero el papel del discurso "experto". Los diferentes profesionales trataron de reconstruir, desde el punto de vista interpretativo propio de ambos campos disciplinares - las ciencias sociales y el derecho-, el contexto de la violación de los derechos humanos, ${ }^{5} \mathrm{y}$ de un modo más amplio del conflicto armado. Más que en una visión crítica de cómo se reconstruyó el contexto de la violación de los derechos humanos, mi perspectiva se centra en el propio contexto de la denuncia y su historicidad. Parto así del presupuesto de que en procesos como las CV las verdades que pueden ser producidas serán siempre parciales, lo mismo que su legitimidad, siendo ésta una cuestión - como veremos - relacionada no tanto con la diversidad de experiencias y subjetividades que las tecnologías del testimonio son capaces de recoger, sino más bien con las relaciones de poder en las que todos los actores están inmersos y que han condicionado el establecimiento de ciertas narrativas $-\mathrm{y}$ sus verdades- como marcos de interpretación dominantes.

\section{VERDAD "JURÍDICA" E "HISTÓRICA": LOS CONTORNOS POLÍTICOS DE UNA POSIBLE CRISIS DE REPRESENTACIÓN}

Es precisamente a partir de las relaciones de poder que marcan el trabajo de las CV que podemos considerar el trabajo de los expertos como una labor destinada a evitar una crisis de representación. Es más, al detenernos en experiencias concretas de este tipo de mecanismos, debemos atender a la existencia

\footnotetext{
${ }^{5} \mathrm{Me}$ distanciaré en este punto del debate entre "legalistas" (quienes defienden que los informes sobre violaciones de los derechos humanos se circunscriban a la información relevante para su constitución como casos jurídicos) y "contextualistas" (que defienden el valor de incluir un mayor proporción de material social y contextual en los informes) (Wilson, 1997: 138). Richard Wilson (2005) ha defendido que el lenguaje globalizado de los derechos humanos, dominado por un aura de cientificismo, tiene una limitada contribución para comprender de un modo más amplio conceptos como la libertad, la ciudadanía o el bien común. Desde esta perspectiva, la pragmática en la que está envuelta el lenguaje jurídico de los derechos humanos, debe abrirse según Wilson a los debates sociológicos más amplios, relativos a la interpretación, comprensión y explicación de las evidencias empíricas y de los límites de su representación (1997: 140). Respecto a los límites del lenguaje legal, véase también el trabajo de Kristen Hastrup (2003) y Fiona Ross (2003b).
} 
de una suerte de conciencia - que permanece generalmente en el nivel de lo implícito- de que su labor debe mostrar la capacidad del aparato científico (del derecho y de las ciencias sociales) de evitar esa crisis: la capacidad de aportar marcos de interpretación científicamente respaldados. Los contornos de esa "amenaza" de una crisis de representación y los elementos sobre los cuales actúa varían dependiendo de cada contexto y de las narrativas que se fueron gestando para interpretar los "hechos de violencia". En el caso peruano, está directamente relacionada con la participación de las comunidades andinas en el conflicto armado y en particular con el proyecto del Partido Comunista del Perú- Sendero Luminoso (PCP-SL) y con cómo este proyecto político ha sido analizado. Así, las relaciones de poder que definieron el contexto de la denuncia desde el inicio del conflicto armado y más concretamente durante el trabajo de la CVR-P, han marcado los modos de entender Sendero, y el triunfo de un marco específico de interpretación de su éxito y fracaso.

Desde el inicio de la década de 1980 el PCP-SL fue pensado como una exterioridad a las comunidades, como un agente cuando menos "insólito" en ese contexto, una idea que se fue gestando ya en las primeras coberturas periodísticas de la región de Ayacucho, principal escenario de la violencia armada. ${ }^{6}$ Por ejemplo, ya en 1981, Máximo González, entonces teniente-alcalde de Cangallo, pequeña capital provincial, muestra a los periodistas de una revista limeña las pintadas con eslóganes maoístas aparecidas en algunos muros de la ciudad, calificadas en el reportaje como "exóticas" en ese contexto, y afirma:

Hay en Cangallo dos centros educativos con 2.200 alumnos del lugar y de una docena de comunidades campesinas. Entre los profesores, hay por lo menos cinco que se dedican a adoctrinar en vez de a enseñar. Les hablan a sus alumnos de marxismo, de muchas cosas por el estilo, los sacan a las calles para pintar paredes con 'consignas revolucionarias' y no podemos hacer nada (Revista Oiga, 23-02-1981, p. 26).

Esta perspectiva se fue afianzando con el tiempo, instaurándose así una narrativa que reflejaba la exteriorización no tanto de la violencia como sobre todo de los elementos político-ideológicos del espacio comunitario andino; se establece así una dicotomía ente el adentro pacífico, apolítico, de las comunidades campesinas vs. el afuera ideologizado y violento de los terroristas (Theidon, 2004; 2006a). En estas narrativas se irá destacando cómo "los militantes y las masas pasaron de ser victimarios a ser víctimas en resistencia, para luego corroer y golpear la estructura política y militar del partido [PCP-SL]" (del Pino, 2007: 6, énfasis añadido). Dentro de este proceso de construcción de determinadas interpretaciones dominantes, se dibuja una división entre actores "armados" y "no armados", siendo la población civil (= el grueso de la población campesina andina) como actor "no armado" la gran categoría referencial, cuya fijación es vital en la reproducción de esas narrativas, de manera que se hace necesario fortalecer los límites que la separan de la categoría de "actores armados".

Cuando un mecanismo como la CVR-P entra en escena, se encuentra así condicionado por esas narrativas instauradas y sus contornos políticos. En este contexto, y como ilustraré en la presente sección, los profesionales asumieron muchas de esas narrativas, las incorporaron a sus informes, pero moderando la reflexión sobre las relaciones de poder que han modelado su producción. Es a partir de este proceso que entiendo que la CVR-P operó como amortiguadora de la "amenaza" de una crisis de representación. En esa operación, los testimonios ocupan un lugar fundamental, el de fuente de legitimidad ético-política de la comisión (Castillejo, 2007: 78). La toma de testimonios fue un proceso central pues ellos conformarían el grueso de la base de datos ${ }^{7}$ de la CVR, que registró 16.917 testimonios individuales y colectivos, participando un total de

\footnotetext{
${ }^{6}$ En el departamento de Ayacucho, región andina situada en el centro-sur del país, se concentró el 40\% de las víctimas reportadas a la CVR-P.

${ }^{7}$ En este trabajo la CVR siguió la metodología del la American Association for the Advancement of Science —la Estimación de Sistemas Múltiples - , que había sido aplicada anteriormente para los casos de Guatemala y Kosovo. Para el caso peruano la base de datos con el número de víctimas y perpetradores se complementó con los datos de la Defensoría del Pueblo (denuncias de desapariciones forzadas), y de varias ONG que llevaban muchos años recogiendo denuncias de violaciones a los derechos humanos. Véase Ball et al. (2000).
} 
18.217 declarantes, de los cuales el $61 \%$ eran familiares próximos a personas muertas o desaparecidas. Los testimonios fueron registrados por los equipos fijos instalados en las oficinas de la comisión y por los equipos móviles que recorrían las comunidades. Para su operacionalización, cada testimonio se traducía en un relato con la información principal (¿quién, cuándo y dónde hizo qué a quién?) relativa a la violación de derechos humanos. Adicionalmente, se desarrollaron investigaciones específicas sobre casos escogidos por su representatividad. Así, entrevistas en profundidad, grupos de discusión y seminarios formaron parte de la Base de Datos Interpretativa (BDI), que en algunos casos fue utilizada como principal fuente para escribir el IF (CVR, 2003, Anexo 6).

Los testimonios se ubicaron en ese espacio discursivo que enfatizaba la labor de la CVR-P como una "oportunidad histórica", un discurso que debe ser entendido como una estrategia política para dar relevancia a un pasado marcado por la tremenda distancia entre los derechos legalmente reconocidos y una realidad de exclusión sufrida por la mayoría de la población (CVR, 2003, Introducción: 30). La CVR-P se pensó como un espacio de reconocimiento de ciudadanía:

No podemos reparar, no podemos ofrecer justicia, pero por lo menos que lo que nos dicen esté bien registrado, tratar a la gente con respeto, como seres humanos, que sí es necesario hacer un reconocimiento. Reconocer que esta parte de la sociedad era más bien ignorada, prescindible, creo que es parte del mensaje de la comisión (Sociólogo, Entrevista de la autora; Lima: septiembre de 2006).

Desde esta perspectiva humanista, los testimonios adquieren un lugar primordial en el proceso de amortiguar la "amenaza" de una crisis de representación. Este trabajo de amortiguación se realiza, desde mi punto de vista, en dos frentes, con importantes consecuencias en la consolidación de un modo de interpretar Sendero. En un frente se asume la interrelación y complementariedad entre la "verdad jurídica" y la "verdad histórica" y por tanto entre el conocimiento legal y el socio-histórico:

Documentos de trabajo del área de esclarecimiento de hechos señalaron en una primera aproximación que su objetivo era la afirmación de la verdad histórica yno de la verdad jurídica. En posteriores discusiones de trabajo ha quedado en claro que éstos no constituyen enfoques antagónicos ni excluyentes, sino más propiamente entradas que contribuyen desde la historia, pero también desde el Derecho, a la reconstrucción y comprensión cabal de la verdad. En este sentido, la reconstrucción histórica de los hechos, en sus contextos (nacional-regionallocal), así como la construcción de narraciones colectivas (historias regionales), se integra con la vertiente de los derechos humanos en donde el esclarecimiento de hechos apelará al empleo de las categorías jurídicas que correspondan. (Centro de información para la Memoria Colectiva y los Derechos Humanos. [SCO38-04; 020412] Presidencia/Área de la CVR/ Esclarecimiento de los hechos; documento: "El enfoque de Derechos Humanos en la labor de esclarecimiento de hechos. Documento de trabajo": sin paginar).

Atravesadas por esta impronta positivista, las discusiones entre los diferentes profesionales en la CVR-P confirman la necesidad de establecer una correa de transmisión directa entre el conocimiento que las metodologías de las ciencias sociales y humanas pueden producir (el esclarecimiento de hechos) y aquel producido desde el derecho (las categorías jurídicas). Esta perspectiva tiene un efecto importante en cómo serán entendidos los testimonios y en cómo serán clasificados los hechos que de ellos son extraídos. Siguiendo lo señalado por Fiona Ross en su análisis de la comisión sudafricana, se puede considerar también que la CVR-P asumió un vínculo incuestionable entre "voz" y "dignidad, y entre "voz" y "ser escuchado":

En el concepto de 'voz' de la Comisión [sudafricana] se asumió la precisión en la comunicación y la claridad en la recepción; la desigualdad de ámbitos sociales y su saturación de poder no lo fueron. (...) Este modelo asumió que aquello que precedió al trabajo de la Comisión fue ausencia de discurso [voicelessness] y silencio respecto al apartheid. (Ross, 2003: 327). 
De modo semejante, en la CVR-P el proceso de toma de testimonios fue asumido como ese momento que posibilitaba "dar voz" a quienes nunca habían podido hablar, lo que a su vez coloca el trabajo de la comisión como un proceso de desestabilización de las relaciones de poder, históricamente producidas, entre Estado y comunidades campesinas andinas:

Obviamente, tomando en cuenta una tradición histórica por la cual nuestro poder judicial siempre ha mostrado una actitud bastante reaccionaria hacia la población campesina. (...) Obviamente que en la CVR se trató con una horizontalidad bien, bien importante, que a mi modo de ver significó mucho el hecho de que la población nos concibiera a nosotros como entes del Estado que les estábamos dando voz a ellas que nunca habian hablado, que nunca habian podido hablar [Antropólogo, integrante de equipos móviles en provincias de Ayacucho, Entrevista de la autora; Huanta: septiembre de 2006].

En un segundo frente de actuación, que operará también como amortiguador de una crisis de representación, la CVR-P asume la tarea de (re)construcción de los actores principales del conflicto bajo la figura de "actores armados", diferenciados y distinguibles de la sociedad civil, con un énfasis particular en la delimitación -desde el campo jurídico de los derechos humanos y del derecho internacional humanitario- del PCP-SL como actor fundamental en el conflicto:

Los organismos de Derechos Humanos (DDHH) a mediados de los 80 cuando conformamos el organismo federal de segundo nivel que es la Coordinadora Nacional de Derechos Humanos, establecimos dos principios: uno, la condena de todo asesinato a sangre fría venga de donde venga, es decir, sin importar quién sea el agente responsable, lo cual significaba condenar los asesinatos que hacía de un lado Sendero Luminoso y condenar los asesinatos a sangre fría que hiciera el Estado. Lo que nos diferenciaba del movimiento de DDHH continental, porque el movimiento de DDHH continental era contra el Estado: usted tiene los organismos chilenos de DDHH y son contra el Estado; los organismo argentinos, ídem, ¿no? (...). Esta historia [la peruana] es diferente, aqui no hay manera de ocultar la cantidad tan importante de crimenes cometidos por grupos subversivos (Doctor en Derecho, entrevista realizada por la autora: noviembre de 2006).

Como ha señalado Richard Wilson (1997: 140141) analizando el proceso de violencia armada en Guatemala, la categoría de "violación de los derechos humanos" se ha constituido sobre la dicotomía entre Estado - como victimario- y sociedad civil —como víctimas inocentes. El discurso legal en la CVR-P tiene, no obstante, otros matices, pues su principal dificultad no fue tanto en relación a la participación del Estado en la violación de derechos humanos, sino en relación a una delimitación clara de la población civil respecto de los senderistas. Así, ya desde los años 1990, el discurso jurídico va a enfatizar la singularidad del conflicto peruano en relación a lo acontecido en el resto de la región latinoamericana pues "por primera vez una fuerza insurgente de izquierda practicó sistemáticamente la violencia contra la población civil igualando, y quizás sobrepasando ${ }^{8}$ a la practicada por el Estado" (Basombrío, 1998: 426). Este proceso de fijación de una frontera entre un actor armado "subversivo" y la población civil es indispensable para que las verdades "jurídica" e "histórica" sean complementarias.

\section{LA POLÍTICA DE LO SILENCIADO Y DE LO IMPLÍCITO}

¿Cómo podemos entender la labor de los expertos en ciencias sociales en estos dos frentes de actuación: la relación entre verdad histórica y jurídica,

\footnotetext{
${ }^{8}$ Esta singularidad fue corroborada en el IF de la CVR: "Para la CVR, el PCP-SL fue el principal perpetrador de crímenes y violaciones de los derechos humanos tomando como medida de ello la cantidad de personas muertas y desaparecidas. Fue responsable del 54 por ciento de las víctimas fatales reportadas a la CVR. Esta cuota tan alta de responsabilidad del PCP-SL es un caso excepcional entre los grupos subversivos de América Latina y una de las singularidades más notorias del proceso que le ha tocado analizar a la CVR" (CVR, 2003, Conclusiones Generales: 317).
} 
y la delimitación de los actores del conflicto? Como ya adelantamos, debemos enmarcar su trabajo a partir de su relación directa con la toma de testimonios y su gestión de los contornos políticos que los forjaron. Entendiendo los testimonios como narrativas sobre el contexto de la denuncia, podemos apreciar su cualidad como relatos construidos a partir de las narrativas instauradas a lo largo de las décadas que duró el conflicto armado, donde se tuvo que gestionar una relación compleja con las autoridades del Estado, reconociendo implícitamente, de parte de la población de las comunidades, su participación en la lucha armada.

Debemos considerar también que los declarantes dieron su testimonio teniendo en cuenta ciertas expectativas respecto a las posibles reparaciones individuales y colectivas, ${ }^{9}$ lo que hace que estos testimonios reflejen, en gran medida, aquello que era posible ser contado así como las formas aceptadas para hacerlo. En este sentido, los testimonios son parte fundamental de los lenguajes políticos plausibles en los contextos posconflicto armado, cuando una gran mayoría de los declarantes (con)viven en "escenarios densos" donde la gente cometió actos de violencia colectiva contra individuos con los cuales habían vivido durante años (Theidon, 2001; 2006a; 2006b). Así, un gran número de testimonios recogidos entre poblaciones de comunidades ayacuchanas muestra que la estrategia de las víctimas y sobre todo de sus familiares fue no sólo suprimir determinados compromisos y discursos políticos de la población en relación con el conflicto armado, sino también su adaptación a esa conceptualización ya establecida del PCP-SL como exterioridad a las comunidades andinas. Se produjo una suerte de revelación estandarizada de un proceso político complejo, cuya traducción en un relato permite la delimitación de los actores y sobre todo de su participación en el conflicto, siendo incorporados en una temporalidad lineal que marca nítidamente el antes y el después del ingreso de "la violencia" y de "la política" en la comunidad. Los siguientes extractos de los relatos de tres testimonios que refieren a lo acontecido en el distrito ayacuchano de Socos son un ejemplo de este procesamiento de las narrativas a través de los testimonios de las víctimas y sus familiares:

Recuerda que en el año de 1981, los senderistas ingresaron a este distrito, reuniendo a los pobladores en la plaza del distrito, donde les hablaron de su partido, luego les pedía que los apoye en sus diferentes acciones (CVR-Sede Huamanga, Relato del Testimonio n ${ }^{\circ}$ 202918).

La declarante refiere que el distrito de Socos era un pueblo tranquilo, pero en 1980 se oían comentarios que en los demás pueblos los miembros de Sendero Luminoso ya habian incursionado; sin embargo, los pobladores del distrito hicieron caso omiso a las habladurías, por el contrario se dedicaban a la agricultura y a la ganadería (CVR-Sede Huamanga, Relato del Testimonio $n^{\circ}$ 202927).

Recuerda que, cuando inicio la subversión, los "terroristas" (militantes de Sendero Luminoso), amenazaban a los hacendados del distrito de Socos, por ello, las autoridades del pueblo, exigieron que se instalara un puesto policial en el distrito para que los protegiera de los "terroristas" (CVR-Sede Huamanga, Relato del Testimonio $n^{\circ}$ 202935).

Estos tres extractos, que considero ejemplares del tipo de narrativas predominantes en los testimonios, constatan que la llegada casi repentina de los senderistas —aquellos que "venían diciendo" a las comunidades - constituyó un momento de ruptura de la vida comunitaria, y que la población fue espectadora de la política de Sendero y que su lealtad final fue para con el Estado. ${ }^{10}$ Podemos vislumbrar cómo la parcialidad de la verdad socio-histórica se compatibilizó con la parcialidad de la verdad jurídi-

\footnotetext{
${ }^{9} \mathrm{La}$ CVR no podía prometer nada y esto debía quedar claro a la hora de relacionarse con los declarantes. En los talleres de preparación destinados a los grupos que iban a recoger los testimonios, se insistió mucho en este aspecto.

${ }^{10} \mathrm{El}$ trabajo etnográfico de Kimberly Theidon en comunidades ayacuchanas ha explorado cómo el "pasado fratricida" ha tenido consecuencias decisivas en las relaciones intra-/intercomunitarias. Una de las estrategias sociales y psicológicas que Theidon encontró de forma reiterada en los relatos de comuneros fue la presencia de la figura del encapuchado ("llegaron los encapuchados") para referirse a los perpetradores como una manera de "mantener algo de distancia y de anonimato allí donde poco de los dos existe" (2006a: 165-166).
} 
ca, permitiendo la separación entre población civil (= víctimas) y actores armados (= Ejército / PCP$\mathrm{SL})$. Es por tanto fundamental comprender que las narrativas imperantes en los testimonios actuaron también en esos dos frentes y facilitaron la amortiguación de una posible crisis en la representación de la violación de los derechos humanos. La "verdad histórica" confluye con la "verdad jurídica" cuando los testimonios recogidos entre las comunidades más afectadas por el conflicto armado confirman la exterioridad de la violencia "subversiva" ${ }_{11}^{11}$ una perspectiva que también apareció como dominante en las interpretaciones ofrecidas en diversas entrevistas por quienes trabajaron en la toma de testimonios en las comunidades:

Yo entiendo y he podido apreciar que el hecho de que la población se haya adherido de alguna forma a Sendero por ese discurso [de justicia social]; se ha dado (...) en las pequeñas ciudades o en poblados. No tanto así en las comunidades. ¿Por qué? Porque en las comunidades, la gente, los campesinos son... casi no tienen mucha instrucción, gente que no sabe leer ni escribir muy bien, que tranquilamente uno va y con cualquier engaño haces que esta persona, bueno, te siguen, pero no porque están bien informados y porque tienen esa ideología. Porque los convencen, o los amenazan. [Abogado, coordinador de equipos móviles en provincias de Ayacucho y Andahuaylas, Entrevista de la autora, Huamanga: septiembre de 2006].

Esta lectura, también paradigmática, de la "inocencia política" de la población campesina, permite reafirmar la delimitación de la población civil (desarmada) como categoría jurídica diferenciada de los actores armados y sobre todo de Sendero.

Es intrínseco a su carácter retrospectivo (Ross, 2001: 252-253) que el testimonio manifieste más sobre el contexto de la denuncia que acerca del conflicto armado, siendo que de este último se revelan verdades "parciales" - el paso "de victimarios a víctimas" - configuradas desde una particular política de lo silenciado (ciertos discursos políticoideológicos) y de lo implícito (la participación en la lucha "subversiva").

\section{TESTIMONIOS, PODER Y CONOCIMIENTO CIENTÍFICO}

Mi apuesta analítica abre diferentes caminos de interpretación del estatus de los testimonios en un proceso político como las CV y del papel de los expertos. En este sentido, parece obvio cuestionarse si la cualidad del testimonio más como revelador del contexto de la denuncia que de los hechos de "la violencia" supone invalidar su facultad como restaurador de dignidad ciudadana, como proveedor de un espacio para contar las violaciones a los derechos humanos. Cabe también preguntarse qué espacio queda, desde esta perspectiva, para el trabajo de los científicos sociales en un proceso como las CV así como en el contexto que las sucede. Una primera respuesta nos llevaría a descentrar la capacidad del discurso experto en la construcción de esa "verdad histórica" y colocarse como una parte más dentro de una variedad de actores y de sus subjetividades políticas (Rodríguez Maeso, 2010). Este descentramiento requiere, desde mi punto de vista, evitar confrontar el discurso experto con la "voz" de los testimonios, una confrontación que era formulada constantemente en las opiniones de muchos de los científicos sociales sobre su labor en la CVR-P, aludiendo generalmente al excesivo protagonismo del discurso científico que favoreció el "desperdicio" de las narrativas recogidas en los testimonios:

Entonces yo tengo la idea de que los testimonios se han desperdiciado. Mejor dicho, hasta ahora los testimonios no se han usado. Eso me parece un tema complicado sobre esa relación entre quienes elaboraron el informe y las personas que vivieron procesos de violencia. Su discurso no aparece (Historiadora; entrevista de la autora; Lima: abril 2006).

\footnotetext{
${ }^{11}$ Como señalan Laplante y Theidon en un trabajo reciente, era evidente que determinado discurso político de la población campesina fue activamente silenciado: “(...) las comunidades rurales se prepararon para la visita de la CVR-P pactando que sólo los "inocentes" podían testificar para no arriesgar ser excluidos de los posibles beneficios compensatorios. Las autoridades alejaron a los trabajadores de la CVR-P de aquellos que habían participado en Sendero Luminoso, e insistieron en que la 'violencia había llegado' de fuera" (2010: 303).
} 
La relación entre las interpretaciones desde la experticia y las "voces" de los testimonios requiere de una fina aritmética; en este sentido, y sin despreciar la relevancia de este dilema, no creo que la cuestión central deba ser planteada en términos de un exceso de interpretación científica que marginaliza las narrativas de los testimonios. Esta perspectiva corre el riesgo de encapsular los testimonios en una retórica positivista que alimenta una "industria de la extracción" y que, paradójicamente, resitúa el discurso experto en un lugar privilegiado en la construcción de conocimiento sobre las "víctimas" (Castillejo, 2005: 50-51), y de modo más general, sobre "lo que pasó". En este sentido, mi propuesta es preguntarnos qué es lo que desde el discurso experto se puede trabajar a partir de las verdades parciales que se producen (y producimos), distanciándome así de la idea de una reconstrucción de la "la verdad histórica" que nos llevarían a interpretar el contexto de la denuncia desde la perspectiva de la "confiabilidad" y "objetividad" de los relatos y testimonios:

La noción de "nunca más" es ostentada en base a la creencia de que un relato confiable $y$ objetivo de las violaciones de los derechos humanos y de la represión, puede ser producido a partir de las memorias y los testimonios de las víctimas de tales violaciones y represiones. Sin embargo, surgen cuestiones sobre en qué medida "la verdad" se encuentra en el hecho de relatarla: si la verdad varía con la forma en que se narra, con las percepciones de aquellos que la cuentan, y de la audiencia que decide lo que es verdad (Schirmer, 2003: 60-61).

Comparto con Jennifer Schirmer que el establecimiento de "la verdad" está sujeto a las relaciones de poder entre quien habla y quien escucha, y defiendo que en un conflicto armado como el peruano, o el guatemalteco desde el cual Schirmer habla, es preciso entender "procesos complejos de lealtades políticas, polaridades, y complicidades que resultaron en extraordinarios niveles de violencia" (ibidem: 63). No obstante, soy escéptica ante su propuesta sobre la inclusión de más voces, más actores, como camino hacia la reconstrucción de "la historia": "Lo que precisamos obtener ahora son testimonios más específicos, más detallados y evidencia de todas las partes del conflicto (...)" (ibidem: 73).
Aun reconociendo la importancia de aportar cuadros más complejos de interpretación de los hechos de la violencia, posiciones como la de Schirmer caen en la visión positivista sobre la obtención de testimonios que prevén un futuro con una verdad completa donde el trabajo de los científicos sociales - en este caso de la antropología y del trabajo etnográfico- sería crucial:

Si la historia final de la verdad depende de con quién eliges hablar -y creer-, entonces necesitamos hablar con muchos, muchos más guatemaltecos antes de tener un sentido completo de la complejidad de la violencia. No podemos asumir que ya sabemos cuál es la verdad, especialmente si esa verdad va a servir como el fundamento político para el futuro de Guatemala (ibidem: 73).

No podemos asumir que sabemos la verdad, pero tampoco podemos asumir que la vamos a saber o más bien, que vamos a conseguir "extraerla". El trabajo experto debe, por el contrario, situarse como uno más dentro de esa política de lo silenciado y de lo implícito, reconociendo que su labor fundamental no es "dar voz" a todas las partes, ni "explicitar" determinadas narrativas, sino entender las relaciones de poder que producen tanto los silencios como las narrativas dominantes, y su legitimidad ético-política:

Teóricamente había que comenzar a trabajar el tema de agencia más allá de cualquier respuesta institucional; cómo la gente comenzó a ser parte de este proceso. Lo otro es la narrativa que construyó la gente y estas narrativas esquivas que pretenden silenciarlas. Hubo una historia que silencia sutilmente otras historias. Entonces, cuál es la historia que nos cuentan y por qué nos las cuentan y esa historia no es necesariamente la historia que pueda darnos lo que pasó realmente. Entonces la agencia no era sólo analizar el hecho, sino en analizar las propias narrativas y cómo se construyen las narrativas. En el hecho ya de la construcción de las narrativas uno podía encontrar también todo este proceso de agencia y el proceso de relación con el Estado (Historiador, entrevista de la autora; Huamanga: abril de 2006). 
Sin embargo, lo cierto es que en un mecanismo político como las $\mathrm{CV}$ el trabajo de los científicos sociales no puede presentarse como una labor de interpretación de las narrativas sino de los "hechos" y su contexto. Así, las comisiones se convierten en una suerte de espacio de mediación donde producir una relación de equilibrio — si bien precario - entre esa "matriz interpretativa preestablecida por el marco teórico-institucional que dirige la investigación" (Castillejo, 2007: 78) y las narrativas de las víctimas, los sobrevivientes y sus familiares. Es más, este equilibro depende, o al menos así se hizo explícito en la comisión peruana, de la legitimidad científica de ese trabajo interpretativo que está, a su vez, íntimamente relacionada con la legitimidad política de las comisiones. En un contexto como el peruano, esta legitimidad política fue siempre muy precaria, un aspecto que puede ser analizado cuestionando a quién se dirigió realmente el trabajo de la CVR-P.

\section{5. ¿A QUIÉN SE DIRIGIÓ LA CVR PERUANA?: LOS LÍMITES DE LA LEGITIMIDAD CIENTÍFICA}

Todos fuimos, en una u otra forma, culpables de esto, desde la tranquilidad de Lima no quisimos enterarnos de lo que pasaba. A todas las víctimas, perdón (Enrique Espinoza, sin fecha; Testimonio de un visitante de la exposición de fotos Yuyanapaq ["Para recordar"]). ${ }^{12}$

Podemos concluir por tanto que la labor del saber experto en la CVR-P consiguió una cierta compatibilización entre la "verdad jurídica" y la "verdad histórica" a partir del tratamiento de una narrativa dominante en los testimonios - sobre todo aquellos recogidos en las comunidades andinas más afectadas por el conflicto armado- que negociaba con lo silenciado y con lo implícito. Esta negociación se dio desde el inicio del conflicto y se fue gestionando durante las dos décadas que precedieron el trabajo de la Comisión. En este contexto, la CVR-P apareció como un proceso ambicioso que tenía como uno de sus principales objetivos — común a todas las $\mathrm{CV}$ - legitimar el conocimiento de la violación a los derechos humanos desde la perspectiva de las "víctimas". No obstante, su propia legitimidad estuvo siempre puesta en causa desde diversos frentes:

(...) sí hubo un enfoque que privilegiaba la interpretación de los hechos y creo que también privilegiaba responder a algunas hipótesis, hipótesis que tienen que ver con temas, por ejemplo, que son preguntas jurídicas: ¿hubo o no crimenes de lesa humanidad?, ¿hubo genocidio?, tales crímenes ifueron cometidos de manera generalizada, sistemática, o son excesos?, ¿quién mató más? Claro, todo el mundo decía: "no, eso no es lo importante, es una pregunta política". Bueno, todas las preguntas eran políticas. Bueno, éticamente todas las vidas son iguales, entonces no importa quién mató más. (...) Si la Comisión dice que fueron igual, entonces es anti no sé qué, si la comisión dice que fue más Sendero, como el Estado ha financiado, no sé qué... y si la Comisión dice al revés entonces son una sarta de comunistas. Entonces ¿cómo sentarse a dar una respuesta que obviamente va a dejar insatisfacciones, como lo ha hecho, a todos? (Sociólogo, Entrevista de la autora; Lima: 2006).

La comisión venía también a competir... la gente tenía ya su propia memoria, entonces en otra lectura lo puedes ver como un espacio de contenciones: ¿por qué vas a venir a hablar de mis muertos? (Historiador, entrevista de la autora; Huamanga: abril de 2006).

Ambas reflexiones hacen evidente que, como ya adelantábamos, la legitimidad científica de la CVRP fue escamoteada por la precariedad de su legitimidad política; si todas las preguntas, todas las hipótesis de trabajo eran políticas, también lo fue, obviamente, el IF y la "verdad" producida en ese relato. Los testimonios - tanto los ofrecidos confidencialmente como en las Audiencias Públicas - fueron parte de las diversas estrategias de las víctimas, de los sobrevivientes, de sus familiares - sobre todo de la población de las comunidades andinas - para po-

\footnotetext{
${ }^{12}$ Testimonio destacado en una de las salas de la exposición en el Museo de la Nación en Lima (visita de la autora en Octubre de 2006).
} 
sicionarse frente al Estado y la "sociedad nacional", que en gran medida los legitimaría como héroes que vencieron al PCP-SL y como víctimas de violaciones de los derechos humanos. Pero para los profesionales que trabajaron en la CVR-P, los testimonios fueron sobre todo los relatos que podían contar y mostrar un escenario de horror y violencia a esa sociedad peruana que no había vivido el conflicto en carne propia, y que no le atribuyó la importancia debida. Es decir, estaríamos ante la construcción de lo que Boltanski ha denominado el "espectador moral": cuando los informes introducen no solamente la descripción del "sufrimiento" del desdichado (le malheureux) sino también la del "espectador que lo observa" (2007: 90-91). Sin embargo, en el IF se denunció, precisamente, la ausencia de un "espectador moral" entre grandes sectores de la sociedad peruana, un aspecto que se explicaría por las "brechas" que separan a las víctimas del resto de población peruana en términos de estatus socio-económico, nivel de educación formal, origen étnico-racial:

Debido al racismo y la subestimación como ciudadanos de aquellas personas de origen indigena, rural y pobre, la muerte de miles de quechua hablantes fue inadvertida en la opinión pública nacional. Su ausencia y el clamor de sus familiares no fueron suficientes para constituir una memoria pública activa e influyente. Durante los años de violencia, resultó más fuerte la distancia que separa a la mayoría de víctimas y al resto de la población peruana (CVR, 2003, Tomo VIII, Capítulo 2: 103).

La CVR-P pretendió conformarse como un espacio para restaurar ese cordón de transmisión entre víctimas, familiares y el Estado, por un lado, y entre víctimas, familiares y conciudadanos, por otro. No obstante, ese cordón de transmisión funcionó sólo parcialmente; o dicho de otro modo, todas las partes se involucraron en la acción de relatar y de recibir/ aprender ese relato desde perspectivas que no cuestionaran ciertas verdades ya negociadas, y que por tanto no cuestionarn ni lo silenciado ni lo implícito. Así, la transmisión hacia la "sociedad nacional" que no vivió el conflicto de la forma en que afectó a las comunidades andinas enfatizó — sobre todo a través de las Audiencias Públicas - una "dignificación moral" de las víctimas, lo que pasaba por su despolitización:

Para dignificar moralmente a las víctimas, éstas son obligadas a parecer puras e inocentes. Aqui es donde el sentimentalismo kitsch juega su papel. Cuando se dirige a los inocentes, la solicitud de respetar a los seres humanos es fácil. Pero la idea de respetar a los seres humanos es más necesaria cuando no son puros y solo parcialmente inocentes (Margalit, 2004: 8).

De este modo, la formación de una "comunidad emocional" mediante la conexión de experiencias individuales que puedan ser apropiadas de manera colectiva (Jimeno, 2007: 187), pasó por el silenciamiento de determinada parte de la historia y por la reificación de "la violencia" como algo opuesto a un presente y un futuro democráticos. En relación al Estado se creó, como hemos visto, una relación precaria donde la CVR-P tuvo que gestionar el contexto de la denuncia. Además, la trayectoria de muchos de los profesionales que trabajaron en la Comisión, marcada por el trabajo académico y en ONGs vinculadas con los derechos humanos y con proyectos de desarrollo local en las comunidades, ha condicionado la incorporación del trabajo de la CVR como parte del Estado y su disponibilidad para dar cuenta de su responsabilidad en la violación a los derechos humanos:

Era una mixtura entre ser una administración pública y ONG, porque eso era la CVR, actuó como ONG (...). Y por qué el discurso de la CVR... una de mis hipótesis es que la CVR nunca fue vista como que hablaba en nombre del Estado; o sea, cuando tú ves la entrega del Informe por Salomón Lerner, ${ }^{13}$ ves la entrega de un ciudadano representante de la sociedad civil o de las ONG, pidiéndole al Estado y no de una relación Estado-Estado (...) (Abogada; entrevista de la Autora; Lima: septiembre de 2006).

${ }^{13}$ Presidente de la CVR-P. 
Podemos concluir por tanto que:

(i) Un mecanismo como las $\mathrm{CV}$ actúa en un espacio político moldeado por las fisuras (históricas) en la relación entre las poblaciones más afectadas por el proceso de violencia armada y el Estado así como en las relaciones estadoestado y entre los ciudadanos.

(ii) Es por tanto evidente que la "verdad histórica" y la "verdad jurídica" serán siempre parciales y en gran medida, así debe ser. Es decir, los dispositivos puestos en funcionamiento por las CV no pueden ejercer como mecanismos de disrupción total de las narrativas ya instauradas aunque sí pueden lograr un escenario de cuestionamiento político y jurídico de la actuación del estado, el aspecto en el cual quizás la CVR-P fue menos exitosa.

(iii) Igualmente, otro proceso recurrente en este tipo de mecanismos políticos es la despolitización de las "víctimas", si bien en un contexto como el peruano ha tenido un cariz diferente que en otros casos como el de la CONADEP y el informe Nunca Más donde esa confrontación jurídica Estado-Estado se convirtió en uno de los principales si no el principal efecto político de la comisión. ${ }^{14}$

Teniendo en cuenta estos tres elementos, creo que es posible apuntar a las diferencias entre las herramientas del conocimiento legal y aquellas de las ciencias sociales. Considero en este sentido que el conocimiento experto desde las ciencias socia- les y sus herramientas metodológicas no tienen en este contexto un valor añadido por su capacidad de "extraer" las posiciones subjetivas sobre lo que pasó, que permitirían poder completar las verdades parciales, en contrapunto con la rigidez del conocimiento legal y su empeño en establecer una relación directa entre "los hechos" y las categorías normativas. Considero más bien que la intervención desde las ciencias sociales tiene la capacidad de evidenciar los límites de su conocimiento y de apuntar hacia otras vías de interpretación del conflicto armado y sobre todo, en el contexto peruano, de reinsertar lo político-ideológico en sus variadas formas, es decir de reinsertar el "viejo" problema de la agencia política. En este sentido, debemos tener en cuenta que la "verdad jurídica" se estableció a partir de ciertos presupuestos interpretativos - sobre todo en relación a la naturaleza de la lucha armada y del PCP$\mathrm{SL}$ - que fueron gestados en gran medida dentro de las ciencias sociales. Y son estos presupuestos los que condicionaron también los contornos de la amenaza de una crisis de representación del conflicto que cuestionarían las dicotomías violencia/democracia y subversión/Estado. Estamos aquí ante una fina línea que separa la búsqueda de otras formas interpretativas y de otros testimonios; mi posición asume que las primeras no requieren necesariamente de los segundos, ni que la conciencia de los límites de las verdades producidas por un mecanismo como las CV resta legitimidad a los testimonios ni capacidad de la restauración/producción de ciudadanía.

\footnotetext{
${ }^{14}$ Sobre la construcción de la figura de "víctimas inocentes" y la narrativa humanitaria en el informe de la CONADEP véase Crenzel (2008: 44-51).12 Testimonio destacado en una de las salas de la exposición en el Museo de la Nación en Lima (visita de la autora en Octubre de 2006).
} 


\section{REFERENCIAS BIBLIOGRAFICAS}

Allen, Jonathan (1999), "Balancing Justice and Social Unity: Political Theory and the idea of a Truth and Reconciliation Commission", University of Toronto Law Journal, 49: 3, 315-353.

Ball, Patrick, Spirer, H. y SpIRer, L. (2000), Making the Case: Investigating Large Scale Human Rights Violations using Information Systems and Data Analysis, AAAS: Washington D.C.

Barahona De Brito, Alexandra (1997), Human Rights and Democratization in Latin America: Uruguay and Chile, Oxford: Oxford University Press.

Basombrío, Carlos (1998), "Sendero Luminoso and Human Rights. A Perverse logic that Captured the Country", in Steve Stern (Ed.), Shining and other Paths. War and Society in Peru, 1980-1995, Durham: Duke University Press.

Boltanski, Luc (1990), L'Amour et la Justice comme compétences. Trois essais de sociologie de l'action, Paris: Éditions Métailié.

Boltanski, Luc (1993/2007), La souffrance à distance, Paris: Gallimard.

Cavallaro, James y Albuja, Sebastián (2008), "The Lost Agenda: Economic Crimes and Truth Commissions in Latin America and Beyond" in Kieran McEvoy y Lorna McGregor (Eds.), Transitional justice from below. Grassroots activism and the struggle for change. Oxford \& Portland, Oregon: Hart Publishing,

Castillejo Cuéllar, Alejandro (2005), "Las texturas del silencio: violencia, memoria y los límites del quehacer antropológico", EMPIRIA. Revista de Metodología de Ciencias Sociales, № 9, enero-junio, 39-59.

CAstillejo Cuéllar, Alejandro (2007), "La globalización del testimonio: historia, silencio endémico y los usos de la palabra", Antípoda, no 4, enero-junio, 76-99.

COMisión de la Verdad y la ReCONCILIACIÓn (2003), Informe Final (www.cverdad.org.pe; último acceso: 3 de septiembre de 2010)

Crenzel, Emilio (2008), La historia política del Nunca Más. La memoria de las desapariciones en la Argentina. Buenos Aires: Siglo XXI.

Degregori, Carlos Iván y Rivera Paz, Carlos (1993) Perú 1980.1993: Fuerzas Armadas, subversión y democracia. Redefinición del papel militar en un contexto de violencia subversiva y colapso del régimen democrático, Lima, IEP.

Degregori, Carlos Iván (2005), "Presentación” en Edilberto Jiménez, Chungui: Violencia y Trazos de Memoria, Lima: COMISEDH.

Del Pino, Ponciano (2007) “Familia, cultura y ‘revolución'. Vida cotidiana en Sendero Luminoso”, en Anne PérotinDumon (dir.), Historizar el pasado vivo en América Latina. www.etica.uahurtado.cl/historizarelpasadovivo/es_ contenido.ph (último acceso: 9 de septiembre de 2009).

HASTRUP, Kristen (2003), "Representing the common good. The limits of legal language" in Richard A. Wilson \& Jon P. Mitchell, Human Rights in Global Perspective. Anthropological studies of rights, claims and entitlements, London: Routledge (ASA Monographs, V. 40), 16-32.

Hayner, Priscilla (2001), Unspeakable Truths. Confronting State Terror and Atrocity. Nueva York: Routledge.

Isbell, Billie Jean (1985/2005), Para defendernos. Ecología y ritual en un pueblo andino, Cuzco: Centro Bartolomé de Las Casas.

JIMENo, Myriam (2007), "Lenguaje, subjetividad y experiencias de violencia”, Antípoda, no 5 (julio-diciembre), 169190.

Laplante, Kisa J. y Theidon, Kimberly (2010), "Commissioning Truth, Constructing Silences. The Peruvian Truth Commission and the Other Truths of 'Terrorists', in Kamari Maxine Clarke; Mark Goodale, Mirrors of Justice. Law and power in the post-Cold War era, New York: Cambridge University Press, 291-315.

LERnER, Salomón (2004), "La verdad, la justicia y los retos de la democracia en el Perú" in La rebelión de la memoria: selección de discursos 2001-2003, Lima: PUCP/IDEHPUCP, 195-218.

MACHER, Sofía (2005), “Audiencias públicas: un espacio para legitimar el testimonio y dignificar a las víctimas del conflicto interno en el Perú" in New Tactics in Human Rights-Centro de Víctimas de la Tortura: Minneapolis. www. newtactics.org./file.php?ID=931 (último acceso: 21 de enero de 2006).

Mamdani, Mahmood (2000), "The Truth According to the TRC" in Ifi Amadiume \& Abdullahi An-Ma'im (Eds.), The Politics of Memory: Truth, Healinf and Social Justice, London: Zed Books, 176-183.

Margalit, Avishai (2004), "Human Dignity between Kitsch and Deification", Litowitz Lecture: Yale University. (www. filosoficas.unam.mx/act_acad/simposio/AMargalit.pdf) (último acceso: 21 de Agosto de 2008). 
Popkin, Margaret; Roht-ArriazA, Naomi (1995), "Truth as Justice: Investigatory Commissions in Latin America”, in Law \& Social Inquiry, 20: 1, 79-116.

Rodríguez Maeso, Silvia (2010), "Comunidades campesinas y la construcción de 'la violencia' en el Perú: secretos, memorias y científicos sociales" in Pablo de Marinis, Gabriel Gatti, Ignacio Irazuzta (Eds.), La comunidad como pretexto. En torno al (re)surgimiento de las solidaridades comunitarias, Barcelona: Anthropos, 281-306.

Ross, Fiona C. (2001), "Speech and Silence. Women's Testimony in the First Five Weeks of Public Hearings of the South African Truth Commission and Reconciliation" inVeena Das, Arthur Kleinman et al. (Eds.), Remaking a World. Violence, Social Suffering, and Recovery, Berkeley: University of California Press: 250-279.

Ross, Fiona C. (2003a), "On having voice and being heard. Some after-effects of Testifying before the South African Truth and Reconciliation Comisión", Anthropological Theory, Vol. 3: 3, 325-341.

Ross, Fiona C. (2003b), "Using rights to measure wrongs. A case study of method and moral in the work of the South African Truth Commission" in Richard A. Wilson \& Jon P. Mitchell, Human Rights in Global Perspective. Anthropological studies of rights, claims and entitlements, London: Routledge (ASA Monographs, V. 40), 163-182.

Rotberg, Robert; Thompson, Dennis (eds.) (2000), Truth vs. Justice. The Morality of Truth Commissions. New Jersey: Princeton University Press.

SChirmer, Jennifer (2003), "Whose Testimony? Whose Truth? Where are the Armed Actors in the Stoll-Menchú Controversy?", Human Rights Quarterly, 25, 60-73.

Steiner, Henry J. (ED.) (1997), "Truth Commissions: a Comparative Assessment. A Transcript of an International Meeting”. World Peace Foundation Reports, 16.

Teitel, Ruti (2000), Transitoinal Justice, Oxford: Oxford University Press.

Theidon, Kimberly (2003), "Disarming the subject: Remembering war and Imagining citizenship in Peru", Cultural Critique, 54, 67-87.

THEIDON, Kimberly (2004), Entre prójimos. El conflicto armado interno y la política de la reconciliación en el Perú, IEP: Lima.

Theidon, Kimberly (2006a), "Los encapuchados: enfrentando el pasado en el Perú" en Elizabeth Jelin \& Susana G. Kaufman (comps.), Subjetividad y figuras de la memoria, Buenos Aires: siglo XXI editores.

Theidon, Kimberly (2006b), "Justice in Transition: The Micropolitics of Reconciliation in Postwar Peru", Journal of Conflict Resolution, 50: 3, 433-457.

WiLson, Richard A. (1997), "Representing Human Rights Violations: Social Contexts and Subjectivities" in Richard Wilson (Ed.), Human Rights, Culture and Context. Anthropological Perspectives, London: Pluto Press, 134-160.

WiLson, Richard A. (2001), The Politics of Truth and Reconciliation in South Africa. Legitimizing the Post-Apartheid State, Cambridge: Cambridge University Press. 